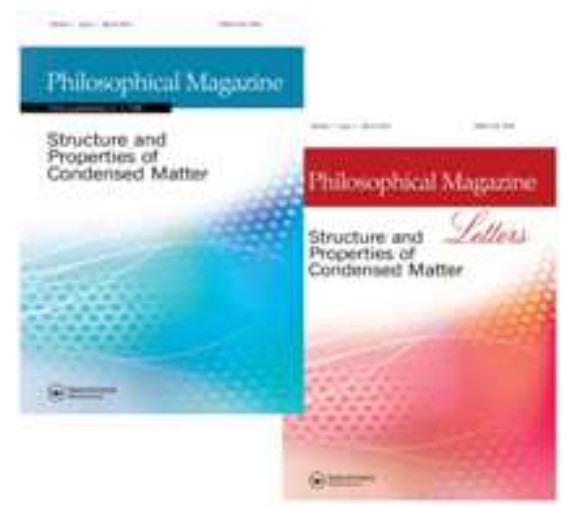

\title{
Determination of the activation energy by stochastic analyses of molecular dynamics simulations of dislocation processes
}

\begin{tabular}{|r|l|}
\hline Journal: & Philosophical Magazine \& Philosophical Magazine Letters \\
\hline Manuscript ID: & TPHM-11-Mar-0099.R1 \\
\hline Journal Selection: & Philosophical Magazine \\
\hline Date Submitted by the \\
Author: & $10-$ May-2011 \\
\hline Complete List of Authors: & Monnet, Ghiath; EDF, MMC \\
\hline Keywords: & $\begin{array}{l}\text { atomistic simulation, dislocation dynamics, molecular dynamics, } \\
\text { thermal activation, statistical physics }\end{array}$ \\
\hline Keywords (user supplied): & \\
\hline \multicolumn{2}{|r}{} \\
\hline
\end{tabular}

\section{SCHOLARONE ${ }^{\text {M }}$ Manuscripts}




\begin{abstract}
In this paper, we investigate the probability and the probability density of thermal activation, of stress-driven dislocation processes, simulated by Molecular Dynamics (MD). Stochastic analyses of the survival probability are found to lead to simple relations between the loading history and the distribution of the interaction time and strength. It is shown that the determination of the activation energy associated to a thermally activated event can be achieved by a reduction of the stochastic process to a process obeying the Poisson's distribution, preserving the activation probability at the survival time. The method is applied to the kink-pair mechanism on screw dislocations in iron. Predictions are compared with experimental results and with other methods reported in the literature, which allows to underline the difference in the approximations and in the assumptions considered in these models.
\end{abstract}

\title{
1. Introduction
}

In the last ten years, the literature witnessed a fast development of Molecular Statics (MS) and Molecular Dynamics (MD) simulations of plastic deformation, for a review see [1,2]. Different mechanisms were simulated: dislocation generation [3,4] and motion [5,6], interactions with point defects [7,8], voids [9], precipitates [10,11,12], dislocation loops [13] and other dislocations $[14,15]$. In all cases, the major contribution of MD simulations was its ability to account for the whole spectrum of atomic vibrations. It is thus a powerful technique for the investigation of effects of the temperature reproducing thermal activation at the atomic level of a large variety of dislocation processes.

Despite the large number of reported investigations, only few analyses were proposed to extract thermal activation parameters. The first approach was the use of the nudged elastic band in MS for the determination of the activation path $[16,17]$. Usually, this method is based

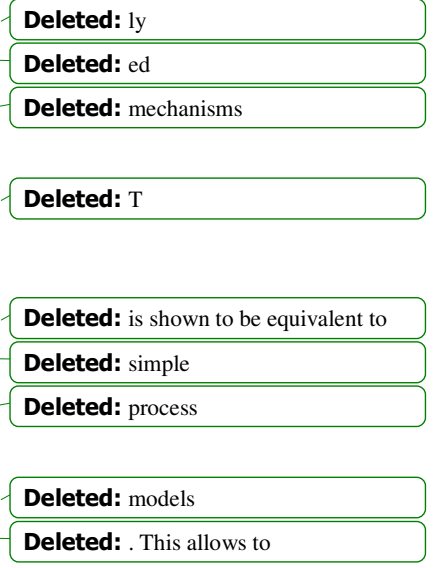

Deleted: i

Deleted: the Deleted: most appropriate

Deleted: and the kinetic energy of atoms 
on the identification of the energetical landscape at zero temperature. The first analysis of thermal activation in MD simulations was reported by Domain et al. [6] who investigated the screw dislocation motion in iron at low temperature. They discussed and explained the origin of the difference between the critical stress measured in experiment and that computed in simulations. Rodney [18] simulated the motion of the edge Lomer dislocation in aluminum and reported a method for the estimation of the activation energy as a function of the average jump stress. Analysis of thermal activation of dislocation nucleation from free surfaces was reported by Zhu et al [3], while Warner et al. [19] investigated temperature effect on dislocation nucleation from a crack tip. Recently, Monnet et al. [20] proposed a method for the extraction of the activation energy based on the concept of the equivalent stress. A priori, these methods look different and their theoretical bases are not clearly connected.

In this paper, we apply the theory of stochastic systems on MD simulations of thermally activated dislocation processes. We show how the thermal activation simulated in MD can be reduced to an equivalent Poisson's process with an equivalent rate and stress. This enables the computation of the activation energy associated to any loading history. The method is compared to other methods reported in the literature. The stochastic analyses allow for the discussion of the assumptions and the approximations of these methods and underline their common features.

The paper is organized as follows. In the next section, we recall some general results of the probability theory applied to thermal activation in connection with the transition theory. Then we discuss the structure of the activation probability and its density in both simulation and laboratory loading conditions. In section 4 , we present a method for the determination of the activation energy based on the construction of an equivalent Poisson's process. Then, in section 5, the method is applied on the case of kink-pair mechanism. Predictions are compared with experimental data and with the predictions of other methods reported in the

\section{General considerations}

\subsection{Probability and the rate function}

Consider a simulation in which several activation events may take place. Let's start observation at a given time $t=0$ and analyze the probability of activation at a given time $t=$ 
$\theta$. We denote $P_{s}(\theta)$ the probability of having exactly $s$ activated events in the interval $[0 ; \theta]$ and $p_{s}\left(t_{1}, \ldots t_{\mathrm{s}}\right)$ the density of probability of having these $s$ events at, respectively $t_{1}, \ldots t_{\mathrm{s}}$, where $t_{i}<\theta$. In the case where the events are undistinguishable, the number of combination of $s$ events being $s$ !, we can express every probability as a function of the probability densities [21,22]:

$P_{s}(\theta)=\frac{1}{s !} \int_{0}^{\theta} p_{s}\left(t_{1}, \cdots, t_{s}\right) d t_{1} \cdots d t_{s}$

The normalization conditions is $\sum_{s=0}^{\infty} P_{s}(\theta)=1$, where $P_{0}(\theta)$ is the probability that no activation event occurs in the considered interval. It is commonly called the survival probability over $[0 ; \theta]$. The average number of activated events is simply given by:

$\langle n\rangle=\sum_{s=1}^{\infty} \frac{1}{(s-1) !} \int_{0}^{\theta} p_{s}\left(t_{1}, \cdots, t_{s}\right) d t_{1} \cdots d t_{s}$.

However, the description in terms of probabilities is that $p_{s}$ are difficult to measure directly

Deleted: The problem with from experiment or to compute in MD simulations. The quantity usually given in these studies is the rate of activation $\omega$, i.e. the frequency at which the activation process is observed. According to the transition theory [23], for stress-assisted thermally activated processes, $\omega$ usually follows an Arrhenius-type equation :

$\omega(t)=v \exp -\frac{\Delta G(\sigma(t))}{k T}$,

where $v$ is a pre-exponential factor of a frequency dimension, $\Delta G(\sigma)$ the activation energy depending on the effective stress $\sigma, k$ the Boltzmann constant $\left(8.610^{-5} \mathrm{eV} / \mathrm{K}\right)$ and $T$ the absolute temperature. It is important to note here that, the quantity $\sigma$ is the local or the effective stress $[6,20]$, which is different from the applied stress when any internal stress source is present, such as free surfaces [6], distortion fields of obstacles [11,8] or simply the self-stress of the dislocation [24], etc. In experiment, the determination of the effective stress is rather a complex task and requires the consideration of sophisticated elastic models [25]. For the sake of simplicity, we refer in the rest of this paper to the effective stress simply by the stress $\sigma_{\text {, }}$

In general, $\omega$ is a function of time since the temperature or the stress may vary during the simulation. This important function provides the property that $\omega(t) d t$ is the infinitesimal probability of activation between $t$ and $t+d t$, regardless of the number of events that may take place outside the interval $d t$. $\omega(t)$ is not a probability density function since it does not accept

Deleted: 20

Deleted: 17

Deleted: We consider $\Delta G$ to be only a function of stress.

Deleted: This function 


\subsection{Survival probability}

In an MD simulation, Either one event can take place [9, 10,28]; or several events can occur $[6,18,29]$. Starting from $t=0$, let us examine the structure of the activation probability at a given time $\theta$ in a system of only one possible activation event. In this case $P_{s}(\theta)$ are zero for all $s$ different from 0 and 1 . For the sake of simplicity the activation probability and the Activation Probability Density (APD) are now written as $P(\theta)$ and $p(\theta)_{\mathbb{v}}$ The resulting normalization condition reads $P(\theta)+P_{0}(\theta)=1$ for all values of $\theta$. The infinitesimal probability $d P(\theta)$ that the activation takes place between $\theta$ and $\theta+d \theta$ and not before is proportional to the probability that the system has survived without activation up to $\theta$, which is $P_{0}(\theta)$, and to the probability of thermal activation in the infinitesimal interval between $\theta$ and $\theta+d \theta$, that is $\omega(\theta) d \theta$. Thus we deduce $d P(\theta)=\omega(\theta) \times P_{0}(\theta) \times d \theta$. Given the normalization

condition on probabilities, one can express the variation of the survival probability as: $d P_{0}(\theta)$ $=-P_{0}(\theta) \times \omega(\theta) \times d \theta$. Consequently, we find:

$$
P_{0}(\theta)=\exp \left[-\int_{0}^{\theta} \omega(t) d t\right] .
$$


Note that, since the probability that nothing occurs must decrease with time, the derivative of the survival probability is always negative. Using the normalization condition, we obtain the APD:

$$
p(\theta)=\omega(\theta) \exp \left[-\int_{0}^{\theta} \omega(t) d t\right]
$$

In principle, every MD simulation provides an estimate of the survival time $\theta_{s}$. If different independent MD simulations are carried out using the same conditions, the average of the survival time measured in MD should, by virtue of the principle of ergodicity, matches with the following formula:

$$
\left\langle\theta_{s}\right\rangle=\int_{0}^{\infty} \theta_{s} \omega\left(\theta_{s}\right) \exp \left[-\int_{0}^{\theta_{s}} \omega(t) d t\right] d \theta_{s} .
$$

$\theta_{S}$ is called the waiting, activation or incubation time. Eq. 8 is the general expression of the average activation time, obtained without any restriction on simulation conditions.

In the following section we examine the structure of the function $p(\theta)$ depending on the loading conditions.

\section{Survival probability in MD}

\subsection{Example of thermally activated process}

In order to illustrate our reasoning in the analyses of MD simulations, we need to restrict our discussion to the good order of magnitude characteristic of atomic scale processes. We choose the example of the thermally activated Kink-Pair (KP) nucleation [30], allowing the motion of dislocations submitted to large lattice friction, This process was widely investigated in MD simulations for simulating the motion of screw dislocation in iron [6], the Lomer dislocation [18] in Al and the edge dislocation in iron gliding on the \{112\} planes [31]. The case of the screw dislocation in iron constitutes a rare example where the activation energy as a function of the stress was determined in a large number of experimental investigations [32,33,34]. These investigations were analyzed by Naamane et al. [35], who underlined the fact that the critical effective stress for the nucleation of a KP should not be confused with the conventional yield stress measured at $0.2 \%$ of deformation. Fitting these data allowed for the determination of $\Delta G(\sigma)$ and the corresponding rate function: 
$\omega(\sigma, T)=H L \exp \left[-\frac{\Delta G_{0}}{k T}\left(1-\sqrt{\frac{\sigma}{\sigma_{0}}}\right)\right]$,

(9)

where, $H=410^{20} \mathrm{~s}^{-1} \mathrm{~m}^{-1}, \Delta G_{0}=0.84 \mathrm{eV}$ and $\sigma_{\mathrm{o}}=360 \mathrm{MPa}$. The rate function is proportional to the dislocation segment length $L$ [36,37]. The parameter $H$ was deduced from experimental results [35] and can also be deduced from theoretical approaches reported by Louchet et al. [36]. Within this approach, $H$ is equal to $\left(v_{D} \times b / l_{c}^{2}\right)$, where $l_{c}$ is the critical length of the kinkpair taken equal to $10 b$. With a typical value of $10^{13} \mathrm{~s}^{-1}$ for the Debye frequency $v_{D}$, and 0.248 $\mathrm{nm}$ for the Burgers vector, one finds a value for $H$ close to $410^{20} \mathrm{~s}^{-1} \mathrm{~m}^{-1}$. Therefore, this value is considered characteristic of the process and independent of the loading conditions.

The numerical values of parameters in Eq. 9 were used in DD simulations [35] and were found to provide a realistic description of the collective behavior of dislocations in good agreement with experiment. Furthermore, the square root dependency on the stress is in agreement with theoretical models of the KP nucleation [38,39].

The average velocity of the dislocation is proportional to the rate function: $v=b \omega\left(\sigma_{R} T\right)$. Since conditions in MD and experiment are quit different, we will distinguish two different sets of conditions: (i) the laboratory (or experimental) conditions in which we consider the following typical values: $L=1 \mu \mathrm{m}, v=1 \mu \mathrm{m} / \mathrm{s}$, a strain rate $=10^{-4} \mathrm{~s}^{-1}$; and (ii) MD conditions [6], where we take: $L=10 \mathrm{~nm}$, a strain rate of $1.5 \times 10^{7} \mathrm{~s}^{-1}$, leading to an average dislocation velocity $v=$ $4 \mathrm{~m} / \mathrm{s}$. Comparing with Eq. 3, the frequency $v$ is evaluated to $410^{12} \mathrm{~s}^{-1}$ in MD conditions and to $410^{14} \mathrm{~s}^{-1}$ in experimental condition.

In this paper, the experimentally determined activation energy in Eq. 9 will be used: (i) to illustrate the difference in stochastic properties of thermal activation in MD and in experiment; (ii) for comparison with the the predictions of the method proposed in this paper. These predictions will be also compared with predictions of other methods reported in the literature.

\subsection{Simulation at constant stress}

If the stress is constant $\sigma=\sigma_{c}$, the treatment shown in $\S 2$ simplifies drastically, since the rate function $\omega_{c}$ is also constant. According to Eq. 7, the APD decreases exponentially with time: $p(\theta)=\omega_{c} \exp \left(-\theta \omega_{c}\right)$, leading to the activation probability $P(\theta)=1-\exp \left(-\omega_{c} \theta\right)$. An important

Deleted: 35
Deleted: 32
Deleted: 36
Deleted: 33

\section{Deleted: 35}

Deleted: 32

Formatted 
property is to be underlined here. When the stress is constant, the average incubation time given in Eq. 8 becomes:

$$
\left\langle\theta_{s}\right\rangle=\int_{0}^{\infty} \theta_{s} \omega_{c} \exp \left(-\omega_{c} \theta_{s}\right) d \theta_{s}=\frac{1}{\omega_{c}} .
$$

This result is obvious, since it is the direct consequence of the definition of the rate function. Using Eqs. 3 and 10, if different activation events are simulated, we can have an estimate of the average activation time $\left\langle\theta_{s}\right\rangle$, which allows us to deduce the activation energy:

$$
\Delta G\left(\sigma_{c}\right)=k T \ln \left(\left\langle\theta_{s}\right\rangle v\right)
$$

As pointed out by Monnet et al. [20], the frequency parameter $v$ should be estimated depending on the nature of the obstacle. In some cases, the evolution of the this parameter with temperature can not be neglected. Recently, Ryu et al. [40] reported a large activation entropy for dislocation nucleation. They connect this entropy to thermal expansion with negligible contribution from vibrational entropy. Warner et al. [19] connected the large temperature effect on the activation energy of dislocation nucleation to the changes in the elastic constants and the staking fault energy.

In the case where several events can be activated in the MD simulation at constant stress, the events are thus random and the activation obeys the Poisson's distribution [21]. This is precisely the case in experiment where a given critical stress is associated with an imposed strain rate.

As we will see later, the case of constant stress will be of interest in establishing the method of determination of the activation parameters in MD simulations.

\subsection{Case of constant stress rate}

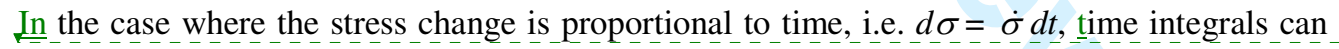
thus be easily transformed to stress integrals [18]. When the stress increases linearly from $\sigma_{\mathrm{S}}$ at $t=0$ to the current value $\sigma_{2}$ Eq. 7 can be rearranged to express the APD per unit stress:

$$
p(\sigma)=\frac{\omega(\sigma)}{\dot{\sigma}} \exp \left[-\frac{1}{\dot{\sigma}} \int_{\sigma_{s}}^{\sigma} \omega(u) d u\right]
$$

From Eq. 12, it can be seen that the APD does not depend only on the current value of stress, but also on the stress rate and on the starting stress $\sigma_{s}$. The profile of $p(\sigma)$ depends on the test conditions. To clarify this feature we calculate $p(\sigma)$ in simulation and laboratory conditions

Deleted: 20
Deleted: 17

Deleted:

Deleted: 21

Deleted: 18

Deleted: Unfortunately, because of

difficulties in carrying out MD simulation at constant stress, the stress is usually not constant. This is the origin of difficulties in determining the activation energies from MD simulations.

\begin{tabular}{l} 
Deleted: The stress increases linearly \\
with time only in two cases: (i) MD \\
simulations are performed at constant \\
stress rate or (ii) MD simulations are \\
performed at constant strain rate but no \\
deformation can be accommodated other \\
than elastically. In this section, we focus \\
on \\
\hline Deleted: 18 \\
\hline Deleted: 16 \\
\hline
\end{tabular}


(see Fig. 1 a and b) with a starting stress $\sigma_{s}=0$. We use the activation energy determined experimentally and discussed in \$3.1. We compute the APD from Eq. 12 in MD and laboratory conditions. With a strain rate of $10^{-4} \mathrm{~s}^{-1}$, the stress rate is close to $7 \mathrm{MPa} / \mathrm{s}$ in experiment, while it is of the order of $10^{12} \mathrm{MPa} / \mathrm{s}$ in MD simulations [6].

Fig. 1: about here.

We plot the ADP profiles for three different temperatures: 10,300 and $600 \mathrm{~K}$ in $\mathrm{MD}$ conditions and three temperatures: 10,100 and $200 \mathrm{~K}$ in experimental conditions. Three general remarks can be made, see Fig. 1. (i) All ADP profiles are peaked around a maximum value of $p(\sigma)$ at a given value of the stress, denoted $\sigma_{p}$. Both $\sigma_{p}$ and $p\left(\sigma_{p}\right)$ vary with the temperature and loading conditions. (ii) The standard deviation of all APD profiles in MD conditions is much larger than in experiment. This explains partially the large fluctuation of the jump stress in MD (see loading curves reported in $[6,18,31])$. (iii) The APD peaks are not symmetrical, hence we can define in addition to most probable stress $\sigma_{p}$ the mean activation stress $\sigma_{m}$, which corresponds to the average of the stress at which the activation occurs, i.e. the average of the maximum stress. By carrying out several independent simulations, the average of the maximum stress provides an estimate of the $\sigma_{m}$ and not of $\sigma_{p} . \sigma_{m}$ and $\sigma_{p}$ are also called the mean jump stress and the most probable jump stress, since the activation is usually the signature of a collective jump of atoms. If the starting stress is zero or very low in all these simulations, we can express the mean jump stress as:

$$
\sigma_{m}=\int_{0}^{\infty} \frac{\sigma}{\dot{\sigma}} \omega(\sigma) \exp \left[-\frac{1}{\dot{\sigma}} \int_{0}^{\sigma} \omega(u) d u\right] d \sigma
$$

Let us examine the values of $\sigma_{m}$ and $\sigma_{p}$ from profiles depicted in Fig. 1. As it can be seen, at $10 \mathrm{~K}$ for all conditions the profile is strongly peaked around the value $\sigma_{\mathrm{o}}=360 \mathrm{MPa}$. The probability to nucleate a KP is thus appreciable only within $2 \mathrm{MPa}$ around the mean jump stress. However, at $600 \mathrm{~K}$ the profile is quite spread with a relatively large variance of almost $80 \mathrm{MPa}$. The average value of the jump stress $\sigma_{m}$ is $168 \mathrm{MPa}$ while the most probable jump stress $\sigma_{p}$ is close to $179 \mathrm{MPa}$. The case of $300 \mathrm{~K}$ is intermediate with $\sigma_{m}=262 \mathrm{MPa}$ and $\sigma_{p}=$ $272 \mathrm{MPa}$. The effect of temperature is clearly more pronounced in experimental conditions. Although the variance remains small for all temperatures $\left(\sigma_{m} \approx \sigma_{p}\right)$, the efficiency of thermal activation is that strong that the average value of the maximum stress at room temperature

\section{Deleted: $\mathrm{t}$}

Deleted: 18

Deleted: 16

Deleted: 31

Deleted: 28
Deleted: It is clear that when $T$ tends to zero, the activation probability tends to the Dirac delta function $\delta\left(\sigma-\sigma_{\mathrm{o}}\right)$. 
tends to zero [35]. While in MD simulations, this athermal plateau cannot be reached. This was first pointed out by Domain et al. [6] who have shown that the difference between experimental and MD simulation conditions provokes a loss of efficiency of the thermal activation in MD. This loss has been evaluated to $21 k T$, which is not negligible compared with $25 k T$, the activation energy measured in experiment.

So far, we investigates the ADP profiles for $\sigma_{s}=0$. Beside the effect of the loading rate on the ADP profiles, the starting stress $\sigma_{s}$ may play an important role. Let us denote $\sigma_{m}$ and $\sigma_{p}$ the values obtained with a zero starting stress. In other cases, the starting stress will be indicated between brackets. As pointed out first by Rodney [18], when $\sigma_{s}$ is small compared to $\sigma_{m}$ say, no significant effect is expected. The reason is that the ADP profiles are peaked around $\sigma_{m}$. But in simulations of kink-pair nucleation $[6,18,31]$, the starting stress for an event $n$ can be very large, depending on the jump stress of event $(n-1)$ (see curves reported in $[6,18,31]$ ). To show the evolution of the ADP profile with $\sigma_{s}$ we plot the ADP profiles in Fig. 2 for different values of $\sigma_{s}: 0, \sigma_{m}$ and $\sigma_{p}$.

Fig. 2: about here.

When $\sigma_{s}=0$, we get the reference values of $\sigma_{m}$ and $\sigma_{p}$. Increasing the value of the starting stress decreases the distribution variance. The profile is not always in the form of a peak, especially when of $\sigma_{s}>\sigma_{p}$. As general tendency, when $\sigma_{s}$ increases, $\sigma_{m}\left(\sigma_{s}\right)$ increases and the difference between $\sigma_{m}\left(\sigma_{s}\right)$ and $\sigma_{p}\left(\sigma_{s}\right)$ decreases. Also, since the stress increment here is proportional to time, we can also note that the average survival time decreases strongly with $\sigma_{s}$. We will see in the following that these features (shown in Fig. 2) are important for the determination of the activation parameters.

It is important to note that when $\sigma_{s}$ is not zero but it remains the same in all the independent MD simulations, the average of the jump stress can be given by:

$\sigma_{m}\left(\sigma_{s}\right)=\int_{\sigma_{s}}^{\infty} \frac{\sigma}{\dot{\sigma}} \omega(\sigma) \exp \left[-\frac{1}{\dot{\sigma}} \int_{\sigma_{s}}^{\sigma} \omega(u) d u\right] d \sigma$

In the case where $\sigma_{s}$ is not the same in the different MD simulations, Eq. 12 should be replaced by:
Deleted: leading to the well know
Deleted: 18

Deleted: 16

\begin{tabular}{l} 
Deleted: 18 \\
Deleted: 16 \\
Deleted: 31 \\
Deleted: 28 \\
Deleted: 18 \\
Deleted: 16 \\
Deleted: 31 \\
\hline Deled: 28
\end{tabular}


where the $\langle\ldots\rangle_{n}$ denotes the average over the number $n$ of independent simulations. In these conditions, it is difficult to predict the average jump stress from MD simulations.

\subsection{Case of constant strain rate}

Simulations at constant strain rate imposes a constant deformation increment to be accommodated at every simulation step. If the dislocation does not move significantly before the activation event, then all the deformation is accommodated elastically and the stress remains proportional to the strain, i.e. to the simulation time. In all other cases, the stress profile can be any profile $\sigma(t)$. Here nothing can be said about the stochastic properties other than the general equations shown in $\S 2.2$. But, as far as we are only concerned with the activation energy $\Delta G(\sigma)$, a possible approach can be used. This is the objective of the next section.

\section{Determination of the activation energy function}

We assume that the energy barrier is independent of the temperature, except for the entropy contribution [23]. Ngan et al. [41] proposed an original approach of the stochastic dislocation nucelation. They compared their prediction of the survival time with MD and experimental results. They considered the Weilbull distribution, which is appropriate in predicting size effect, but still an empirical approach, that cannot be connected to physical parameters. We do not discuss this approach here since no use of the transition theory was made.

In this section we will see that any stress-assisted activation process can be in general reduced to a Poisson's process with a well defined stress and activation energy. First we show how this can be done on the level of one activated event and, then, we generalize the treatment in order to consider a set of separate activation events. In all these cases we determine the constant stress leading to an equivalent Poisson's process.
Deleted: In MD simulations, the applied stress is computed at every time step $\sigma_{a p p}(t)$. Here we should note that in many cases the applied stress is not the only component experienced by the dislocation. For example, the dislocation feels free surfaces [6] or precipitate stress field [11]. In addition, in the case of local obstacles, the dislocation bows-out before unpinning [17], which generate on the pinned segment a strong self-stress component due to the dislocation line component due to the dislocation line
tension. In these cases, the quantity of interest is not $\sigma_{a p p}(t)$ but the effective (or local) [17] stress which is by definition the simple sum of these components. For the sake of simplicity in the following we denote this stress by $\sigma(t)$. II The characteristic quantity of interest for every obstacle is the activation energy $\Delta G$ as a function of the local or effective stress.

Deleted: 23

Deleted: 20

Deleted: generation 
The idea proposed in this paper is based on the reduction of the complex activation process with $\sigma(t)$ to a Poisson's process whith a constant stress $\sigma \equiv \sigma_{\mathrm{c}}$. We can call this stress the Poisson's stress since it represents the constant stress making the associated Poisson's process equivalent to that observed with the "real" profile $\sigma(t)$.

In order to identify the equivalent Poisson process, we need first to identify $\omega_{c}$ and $\sigma_{c}$ from the activation time and the stress profile $\sigma(t)$ computed in the MD simulation. $\underline{\sigma}_{c}$ can be computed by imposing that the activation probability $P\left(\theta_{s}\right)_{o}(t)$ is the same as the activation probability of the equivalent Poisson's process $P\left(\theta_{s}\right)_{\sigma=\sigma_{c}}$. This is schematized in Fig. 3b. Since, the stress is constant in the Poisson's distribution, this condition can be expressed as:

$\exp \left(-\omega_{c} \theta_{s}\right)=\exp \left[-\int_{0}^{\theta_{s}} \omega(t) d t\right]$.

The solution of Eq. 16 is simply:

$\omega_{c}=\frac{1}{\theta_{s}} \int_{0}^{\theta_{s}} \omega(t) d t$

The rate of the equivalent Poisson's process equals thus the average over the survival time $\theta_{s}$ of the rate function: $\omega_{c}=\langle\omega(t)\rangle$. It is important to note that this result is obtained without any approximation or restriction on the shape of the function $\sigma(t)$. Of coarse, the two profiles of the APD are quit different (see Fig. 3b); the condition in Eq. 17 imposes only that the time integration of the two ADP gives the same value at $t=\theta_{s}$.

In general, the activation energy is not known. MD simulations provide $\sigma(t)$ and the survival time $\theta_{s}$. The question now is how to determine the Poisson's stress $\sigma_{c}$ directly from the $\sigma(t)$. In order to answer this question we can use the following approximation $[6,20,31]$. It consists in

Deleted: ere

Deleted: is constant and equals

Deleted: The interest of the Poisson's distribution is that all stochastic properties are known, enabling the determination of the sought function $\Delta G\left(\sigma_{\mathrm{c}}\right)$. It is a one-parameter distribution, corresponding in our case to the rate function $\omega_{c}$ or the associated stress $\sigma_{c}$.

Deleted: The appropriate equivalence can be readily obtained

Formatted

Deleted: at the activation time $\theta_{s}$

Deleted: in

Deleted: density or the

\begin{tabular}{l} 
Deleted: 20 \\
Deleted: 17 \\
Deleted: 31 \\
Deleted: 28 \\
\hline
\end{tabular}


the expansion of the activation energy to the first order around the value of $\sigma_{e}: \Delta G=A-V \sigma$, where $A$ is constant and $V$ the activation volume. This enables us to reduce Eq. 17 to the following expression:

$$
\sigma_{c}=\frac{k T}{V} \ln \left\langle\exp \left(\frac{V \sigma(t)}{k T}\right)\right\rangle .
$$

Eq. 18 is valid for all stress profiles computed in MD. As pointed out in [20], in the special case where the stress is proportional to time and the starting stress is zero, Eq. 17 simplifies to:

$$
\sigma_{c}=\sigma_{e}-\frac{k T}{V} \ln \frac{V \sigma_{e}}{k T}
$$

Eq 18 or 19 can be easily used to determine the Poisson's stress, using a rough estimation of the activation volume. As can be guessed from Eq. 19, the difference between $\sigma_{c}$ and the maximum stress $\sigma_{e}$ depends rather on the temperature than on the slightly varying activation volume. In the next section, we give an evidence to this postulate using experimental data. It can also be seen from Eq. 19 that when the temperature goes to zero, the Poisson's stress tends to the maximum stress, whatever the value of the activation volume.

\subsection{Generalization to many activation events}

In MD simulations, the activation event cannot be considered as independent. Besides, loading conditions during the activation of a given event, i.e. the $\sigma(t)$ profiles, may be different from one event to another one. Following the method described in § 4.1., one can associate a critical stress to every event, which are not necessary the same. Although, every event was reduced to a Poisson's process, the ensemble of events can not be considered as belonging to one Poisson's process.

Let us consider $N$ events simulated in one MD simulation or in several independent MD simulations. We can always associate to an event $n$ an activation time $\theta_{n}$, a Poisson's stress $\sigma_{c, n}$ and rate $\omega_{c, n}$ using the procedure presented in $\S$ 4.1. In this framework, we cannot deal with avalanches of activated events. Although the origin of these avalanches was explained [31], the correlation between them is still difficult to couple with thermal activation kinetics. Poisson's stresses $\sigma_{c, n}$ are called "partial" Poisson's stresses, since they account for only one event at a time. For every event, the activation probability at the survival time is given by: $P_{n}\left(\theta_{n}\right)=1-\exp \left(-\theta_{n} \omega_{c, n}\right)$. 
Now, we seek the Poisson's process of the whole ensemble of the events. In practice, we seek the Poisson's stress $\sigma_{c}$ as a function of the different $\sigma_{c, n}$ and their associated survival times $\theta_{n}$. Since, the application of the $\sigma_{c, n}$ during the $\theta_{n}$ produces the activation of $N$ events, the equivalent Poisson's stress $\sigma_{c}$ should lead to the activation of $N$ events during the interval $\theta_{t o t}=\Sigma\left(\theta_{n}\right)$. For this equivalent Poisson's process, the average activation time $\underline{\theta}_{c}$ is simply the $_{\text {e }}$ average of the computed $\theta_{n}: \theta_{c}=\theta_{t o t} / N=<\theta_{\underline{n}} \geq$. Its rate function $\omega_{c}$ equals thus the inverse of the average survival time $\theta_{c}$, i.e. $\omega_{c}=1 / \theta_{c}$ and the associated the activation probability at $\theta_{c}$ is $P_{c}\left(\theta_{c}\right)=1-\exp \left(-\theta_{c} \omega_{c}\right)$. Since the Poisson's stress $\sigma_{c}$ should in average produce the same activation probability as that produced by the $\sigma_{c, n}$ one may write the necessary condition over $\sigma_{c}$ as:

$\exp \left(-\omega_{c} \theta_{c}\right)=\frac{1}{N} \sum_{N} \exp \left(-\omega_{c, n} \theta_{n}\right)$.

Equation 21, although rigorous, cannot be solved in $\sigma_{c}$ as was solved eq. 16, because events are not independent. A good approximation of Eq. 20 is possible thanks to the special structure of the function $P_{n}$. The probability of activation $P$ after an incubation time $\theta$ in a Poissonian process is given by $1-\exp (-\omega \theta)$, where $\omega$ is the rate associated with the stress $\sigma$ by Eq. 3. Let us examine the variation of $P$ with $\sigma$. The first derivative of $P$ with respect to $\sigma$ is always positive, while the second derivative vanishes at $\sigma=\sigma_{c}$, i.e. when $\Delta G\left(\sigma_{c}\right)=-(k T / V)$ $\ln \left(v \theta_{n}\right)$. When $\sigma$ varies around $\sigma_{c}: \sigma=\sigma_{c}+\Delta \sigma, P$ varies roughly proportionally with $\Delta \sigma$. In Fig. 4, we plot for typical values of the different variables $(L=10 \mathrm{~nm})$ the evolution of $P$ as a function of $\Delta \sigma$. We can clearly see that $P$ varies almost linearly with $\Delta \sigma$ for a wide range of the activation probability (from 0.1 up to 0.9 ).

Fig. 4: about here.

The first derivative at $\sigma_{c}$ is simply $V /(k T e)$, which is independent of $\theta_{n}$ and the value of $\sigma_{c}$ varies with the logarithm of the incubation time (see eq. 11), i.e. $\sigma_{c}$ does not vary strongly with $\theta_{c \text {. }}$

Now we go back to Eq. 20 and we try to take advantage of (i) and (ii). It is easy to find that we can write every activation probability in eq. 21 as :

$P_{n}\left(\sigma_{c, n}\right)=P_{c}\left(\sigma_{c}\right)+\frac{V}{k T}\left(\sigma_{c, n}-\sigma_{c}\right)$
Formatted

Deleted: the sum of the probabilities $P_{n}\left(\theta_{n}\right)$ must match with $N$ times the activation probability characteristic of the Poisson's process $P_{c}\left(\theta_{c}\right)$. With Eq. 20, this condition leads to the equation:

Deleted: in the same way we 
Inserting Eq. 22 into Eq. 21 yields the following solution for the Poisson's stress :

Consequently, the survival times $\theta_{n}$ do not contribute in the determination of the Poisson's stress. The later is simply the linear average of the partial Poisson's stresses $\sigma_{c, n}$. Once the Poisson stress for all events is determined, we can reduce the ensemble of the $n$ events to one Poisson process with a constant stress $\sigma_{c}$. The associated activation energy can be easily determined using the procedure discussed in $\$ 3.2$.

\section{Application to the kink-pair mechanism on the screw dislocation in iron.}

\subsection{Effect of the activation volume}

In the method presented in this paper the activation volume $V$ is required for the determination of $\sigma_{c, n}$. We must thus first check that the computed values $\sigma_{c, n}$ are not strongly affected by the choice of $V$. To do so, we can use the experimentally determined activation rate is Eq. 9 discussed in $\$ 3.1$. We consider a typical mechanical loading condition in MD (see $\S$ 3.3.). The stress is increased from 0 at constant stress rate $d \sigma=\dot{\sigma} d t$, where $\dot{\sigma}$ is of the order of $10^{12} \mathrm{MPa} / \mathrm{s}$. The stress jncreases to a maximum value (the jump stress) when the activation occurs. The average value of the jump stress $\sigma_{m}$ can be determined using eq. 13. In order to calculate the associated Poisson's stress $\sigma_{c}$ from eq. 18, we consider two values for the activation volume: $20 \mathrm{~b}^{3}$, found in experiment at high stresses [33 34]; and $10 \mathrm{~b}^{3}$. The results are depicted in Fig. 5. In order to reveal the effect of imposing a constant value of $V$, we consider another test by deriving the activation energy found in experiment (Eq. 9) to deduce the exact current value of $V$ at the current stress value. We have thus three different values of $V$ for the determination of the Poisson's stress All these estimates are plotted in Fig. 5. As we can clearly see, there is no significant effect of the exact value of $V$ on the computation of the Poisson's stress. We can thus trust the robustness of the value of the critical stress even with a rough estimate of the activation volume.

Fig. 5: about here.

Deleted: As discussed before, $\mathrm{t}$ Deleted: should 
From Fig. 6, we can see that, depending on the starting stress of every event, the APD profile varies from one event to the other. Thee profiles are quite different from the profile obtained with a zero starting stress (see Fig. 1). For some events it is even not possible to distinguish a maximum of the APD. The integration of these profiles furnishes the activation probabilities obtained according to experimental data. In the next section, they will be compared to the activation probabilities deduced from the method proposed in this paper.

\subsection{Determination of the activation parameters}

For every event recorded on the stress strain curve in Fig. 6 we compute the corresponding Poisson's stress $\sigma_{c, n}$ using eq. 18 (see Fig. 7) and a value of $20 b^{3}$ for the activation volume.

We recall here that every $\sigma_{c, n}$ is a constant stress characteristic of Event $n$. The $\sigma_{c, n}$ appear as horizontal line segments in the figure. For every event, $\sigma_{c, n}$ is located between the starting and the maximal stress, but remain closer to the maximum stress. Some domains of integration of eq. 18 are delimited by vertical dashed lines in the figure.

Fig. 7: about here. 
The $\sigma_{c, n}$ vary substantially from one to another event. The Poisson's stress $\sigma_{c}$ amounts to 255 $\mathrm{MPa}$ and shown as a double-line in the figure. From Fig. 7 we can evaluate the average activation time, since simulation reported in [6] is performed at constant strain rate of $1.5 \times 10^{7}$ $\mathrm{s}^{-1}$. $\theta_{c}$ is found close to $0.038 \mathrm{~ns}$. Eq. 11 can then be used to deduce the corresponding activation energy, which yields $\Delta G=0.13 \mathrm{eV}$. The value of $\Delta G$ is therefore associated to the Poisson's stress $\sigma_{c}=255 \mathrm{MPa}$.

\subsection{Validation and comparison with experiment}

The result of the application of the method presented in this paper is $\Delta G\left(\sigma_{c}=255 \mathrm{MPa}\right)=$ $0.13 \mathrm{eV}$. The value can be compared with the experimental data discussed in §3.1. Numerical application shows that for the same effective stress, $\Delta G$ is equal to $0.133 \mathrm{eV}$ which is almost the same as the activation energy obtained from Eq. 11. This is a first and direct validation.

Fig. 8: about here.

The method can also be checked at the level of individual events, by comparing the activation probabilities. Since the activation energy and the Poisson's partial stresses $\sigma_{c, n}$ are now known (see Fig. 7), we can compute the activation probability for every event $P_{n}=1-\exp \left(-\omega_{c, n} \theta_{n}\right)$ and compare it with the activation probability computed form experiment (see Fig. 6). Fig. 8 shows profiles of the activation probabilities computed from experiment (thick line) and from the method presented in this paper (thin line). For every enent, the evolution of the activation probabilities are different, but the average of $P_{n}\left(\theta_{n}\right)$ is almost the same in both approaches: 0.52 and 0.54 . This agreement is noticeable since the $\Delta G\left(\sigma_{c}\right)$ predicted from our stochastic analysis is obtained from a completely different and independent way compared to experiment [35]. 


\subsection{Discussion}

We first discuss the approach proposed by Domain et al [6] and used by Monnet et al [20]. The difference with the method proposed in this paper is the solution of Eq. 21. The solution considered in these works is based on the following reasoning. Since, for every event, the distribution is Poissonian, the different products $\omega_{n} \theta_{n}$ should not be very different from 1 . The Taylor series to the first order in the vicinity of $(-1)$ of the survival probabilities is expressed by:

$\exp \left(-\omega_{i} \theta_{i}\right)=e^{-1}+e^{-1}\left(1-\omega_{i} \theta_{i}\right)$

Inserting Eq. 22 into Eq. 21, we get:

$\omega_{c}=\frac{1}{\theta_{t o t}} \sum_{n} \omega_{c, n} \theta_{c}$

If we expand the activation energy as before $(\Delta G=A-V \sigma)$, we obtain the first order solution of eq. 21 called $\sigma_{\text {ind }}$ (the index "ind" refers to independent):

$$
\sigma_{\text {ind }}=\frac{k T}{V} \ln \left\langle\theta_{n} \exp \left(\frac{V}{k T} \sigma_{c, n}\right)\right\rangle_{N}
$$

The symbols $\langle\ldots\rangle_{N}$ denotes the average over all the $N$ events. Replacing the $\sigma_{c, n}$ by their expression in Eq. 18, one gets:

$$
\sigma_{\text {ind }}=\frac{k T}{V} \ln \left\langle\exp \left(\frac{V \sigma(t)}{k T}\right)\right\rangle_{\theta_{\text {tot }}}
$$

Eq. 27 is the same as Eq. 3 in [6] and Eq. 11 in [20]. It corresponds to the extension of the average in Eq. 18 over all the activation events. Since Eq. 18 was established for a Poisson's process, the solution given in Eq. 27 stipulates implicitly that all events are random and independent. In other terms, there is a competition between these events at any time interval. The events are of coarse not independent, their occurrence obeys conditional probability and not statistics of random independent events. Although, this assumption is not justified from a theoretical point of view, $\sigma_{\text {ind }}$ still provides a good estimate of the Poisson's stress, since the products $\omega_{n} \theta_{n}$ as well as $\omega_{c} \theta_{c}$ are in deed close to 1 . As can be seen in Fig. 7, $\sigma_{c}$ is equal to $255 \mathrm{MPa}$ and $\sigma_{\text {ind }}$ is $266 \mathrm{MPa}$. The difference amounts to $4 \%$. This confirms that the approximation of independent events used in [6,20] provides a good estimate of the Poisson's stress.

In the literature, we can find another method proposed by Rodney [18] and consists in tow approximations furnishing the activation energy and the corresponding stress. (i) The

\begin{tabular}{l} 
Deleted: 20 \\
Deleted: 17 \\
\hline
\end{tabular}


activation energy of the process is considered equal to the effective activation enthalpy $\Delta H^{*}$, defined by:

$\Delta H^{*}=k T \ln \left(\frac{v \Delta \sigma}{\dot{\sigma}}\right)$,

where $\Delta \sigma$ is the stress drop following the nucleation of the KP. (ii) The identification of the associated stress is based on the following reasoning. Simulation of different activation events allows for the determination of the average jump stress $\sigma_{m}$. Instead of using Eq. 13 to connect $\sigma_{m}$ to the APD profile, $\sigma_{m}$ is considered to match with the most probable stress $\sigma_{p}$, obtained at a constant stress rate $\dot{\sigma}$ with a low starting stress. In these conditions, $\sigma_{p}$ is obtained by maximizing the APD. The derivative of the APD in eq. 12 vanishes when:

$\left.\frac{\partial \omega(\sigma)}{\partial \sigma}\right|_{\sigma_{p}}=\frac{\left(\omega\left(\sigma_{p}\right)\right)^{2}}{\dot{\sigma}}$

Together with Eq. 3, Rodney [18] finds:

$-\left.\frac{\partial \Delta G}{\partial \sigma}\right|_{\sigma_{p}}=\frac{k T}{\dot{\sigma}} \omega\left(\sigma_{p}\right)$

The important feature in Eq. 30 is that $\sigma_{p}$ is independent of the starting stress. The computed $\sigma_{p}$ is always larger than $\sigma_{m}$ but remains close to $\sigma_{m}$ (see Fig. 1). However, in the case where the starting stress $\sigma_{s}$ is enough large, the values of $\sigma_{m}\left(\sigma_{s}\right)$ and $\sigma_{p}\left(\sigma_{s}\right)$ can be strongly affected. Fig. 2 and Fig. 6 provide an evidence to this. The profiles of the APD are not always peaked around the fixed value $\sigma_{p}$. Despite this feature, the average of the jump stress remains indeed close to $\sigma_{p}$ because the large values of $\sigma_{s}$ increase $\sigma_{m}\left(\sigma_{s}\right)$ to approach $\sigma_{p}$. (see Fig. 2). We expect the average of the jump stress to be a good estimate of $\sigma_{p}$. The two assumptions considered in this method come to approximate the stress-strain curve, such as that shown in Fig. 6, by a "sawtooth" shape curve, in which, at every activation event, the stress falls down from $\sigma_{m}$ to $\sigma_{m}-\Delta \sigma$, before increasing again up to $\sigma_{m}$. We notice that the survival time $\left\langle\theta_{s}>\right.$ in this approximation can be deduced from the relation $\Delta \sigma=\left\langle\theta_{s}\right\rangle \dot{\sigma}$. We can easily see that Eq. 28 is an approximation of Eq. 11. In the given example of Fig. $6, \Delta \sigma=30 \mathrm{MPa}$ and $\dot{\sigma}=$ $1.1 \times 10^{18} \mathrm{~Pa} / \mathrm{s}$. Numerical application provides the value of $0.122 \mathrm{eV}$ for the activation energy, while the average of the jump stress amounts to $266 \mathrm{MPa}$. These values are in good agreement with experimental results [35]. Although simple and accurate, the method proposed in [18] can be used only when the stress increases linearly with time during the incubation time.

Deleted: 35

Deleted: 32

Deleted: 18

Deleted: 16

Deleted: 17]. 


\section{Acknowledgement}

This work is partially supported by the European project FP7 Project PERFORM60. Details on this project can be found on www.PERFORM60.net.

\section{References}

[1] Bacon D.J., Osetsky Yu.N., Rodney D. in Dislocations in Solids, Vol. 15, J.P. Hirth and L. Kubin eds., Elsevier, Amsterdam, North Holland (October 27, 2009) 
[2] Bulatov VV, Cai W. In: Computer simulations of dislocations. Oxford: Oxford University Press; 2006

[3] Zhu T., Li J., Samanta A., Leach A., Gall K., Phys. Rev. Lett 100, (2008) 025502

[4] Rabkin E., Nam H.S., Srolovitz D.J., Acta Mater. 55, (2007) 2085.

[5] Osetsky Yu. N., Bacon D.J., Model. Simul. Mater. Sci. Eng. 11 (2003) 427.

[6] Domain C., Monnet G., Phys. Rev. Lett. 95 (2005) 215506.

[7] Clouet E., Acta Mater 54 (2006) 3543

[8] Tapasa K., Osetsky Y., Bacon D.J., Acta Mater 55 (2007) 93

[9] Osetsky Yu. N., Bacon D.J., Mohles V., Phil. Mag. 83 (2003) 3623.

[10] Terentyev D.A., Bonny G., Malerba L., Acta Mater. 56 (2008) 3229

[11] Hu S.Y., Schmauder S., Chen L.Q., Phys. Stat. Sol. (b) 220, (2000) 845

[12] Kohler C., Kizler P., Schmauder S., Modelling Simul. Mater. Sci. Eng. 13 (2005) 35

[13] Terentyev D., Grammatikopoulos P., Bacon D.J., Osetsky Yu. N., Acta Mat. 56 (2008) 5034

[14] Rodney D., Phillips R., Phys. Rev. Lett. 82, (1999) 1704

[15] Madec R., Devincre B., Kubin L., Hoc T., Rodney D., Science 301, (2003) 1879

[16] Rasmussen T, Jacobsen1 K. W., Leffers T., Pedersen O. B., Srinivasan S. G. and Jónsson H., Phys. Rev. Lett. 79, (1997) 3676-3679.

[17] Wen M. and Ngan A. H. W., Acta Mater. 48 (2000) 4255-4265

[18] Rodney D., Phys. Rev. B 76 (2007) 144108.

[19] Warner D.H., Curtin W.A., Acta Mater. 57 (2009) 4267-4277

[20] Monnet G., Osetsky Y., Bacon D.J, Phil. Mag. 2009, Phil. Mag. 90 (2010) 1001

[21] Kinney J.J., in Probability - an Introduction with Statistical Applications, John Wiley \& Sons, inc, 1997.

[22] Kampen N. Van, Stochastic Processes in Physics and Chemistry, North-Holland, Amsterdam, 1992.

[23] Vineyard G., J. Phys. Chem. Solids 3 (1957) 121.

[24] Monnet G., Acta Materialia, 55 (2007) 5081 - 5088

[25] Mason J. K., Lund A. C., Schuh C. A., Phys. Rev. B, 73, (2006) 054102.

[26] Caillard D., Martin J.L., Thermally activated mechanisms in crystal plasticity, Pergamon Materials Series Vol. 8, 2003

[27] Kocks U.F., Argon A.S. and Ashby M.F., Prog. Mater. Sci. 19 (1975) 303.

[28] Terentyev D., Bacon D.J., Osetsky Yu.N., J. Phys.: Condens. Matter 20 (2008) 445007 (11pp)

[29] Chaussidon J., Fivel M., Rodnay D., Acta Mater 54 (2006) 3407-3416

[30] Seeger A. and Schiller P., Acta Metall. 10, (1962) 348.

[31] Monnet G., Terentyev D., Acta Mat. 57 (2009) 1416

[32] Quesnel D. J., Sato A., Meshii M., Mater. Sci. Eng., 18, (1975) 199

[33] Kuramoto E., Aono A., Kitajima K., Scripta Metall., 13, (1979) 1039

[34] Spitzig W.A., Keh A. S., Acta Metall., 18, (1970) 611, Acta Metall. 18, (1970) 1021

[35] Naamane S., Monnet G., Devincre B., Int. J. Plasticity 26 (2010) 84

[36] Kubin L.P., Louchet F., Proc. Fifth Int. Conf. High Voltage Electron Microscopy, Kyoto, 1977

[37] Louchet, F., Kubin, L.P., Phys. Stat. Sol. A 56, (1979) 169.

[38] Schoeck, G., Nabarro, F.R.N., 1980. Dislocations in Solids, vol. 2. Amsterdam, North Holland, p. 67.

[39] Hirth, J.P., Lothe, L., 1982. Theory of Dislocations, second ed. Wiley Inter-Science, New York.

[40] Ryu S., Kang K. and Cai W., Proc. Nat. Acad. Sci., 108 (2011) 5174. 
| [41] Ngan A.H., Zuo L, Wo P.C., Proc. R. Soc. A 462 (2006 ) 1661. 


\section{Figures}

Fig. 1: profiles of the activation probability density at different temperatures per stress unit of MPa in (a) MD conditions and (b) laboratory conditions, calculated from Eq. 12, using a zero starting stress.

Fig. 2: effect of the starting stress on the activation probability density per stress unit of MPa, computed using Eq. 12

Fig. 3: (a) a schematic stress profile and the corresponding Poisson's stress; (b) the probability activation for the two stress profiles in (a).

Fig. 4: evolution of the activation probability in the vicinity of the Poisson's stress.

Fig. 5: evolution of the average jump stress (full circles) and the Poisson's stress calculated from experimental data. Open symbols designate values of the critical stress obtained with a constant volume approximation, for squares $\mathrm{V}=20 \mathrm{~b} 3$ and for rectangles $\mathrm{V}=10 \mathrm{~b} 3$. Full triangles refer to the Poisson's stress computed with values of the activation volume derived from experimental data.

Fig. 6: evolution of the stress and the activation probability density as a function of strain in MD simulations of screw dislocation moving in iron at $300 \mathrm{~K}$ and $1.510^{7} \mathrm{~s}^{-1}$ strain rate.

Fig. 7: the stress-strain curve reported in [6]. The straight horizontal segments indicate the Poisson's partial stresses and the double-line the Poisson's stress. The simple horizontal line show the value of the Poisson's stress in the independent-event approximation.

Fig. 8: comparison between of activation probabilities computed from experimental data (thick line) with those obtained using the method proposed in this paper and based on the concept of the Poisson's stress (thin line). 

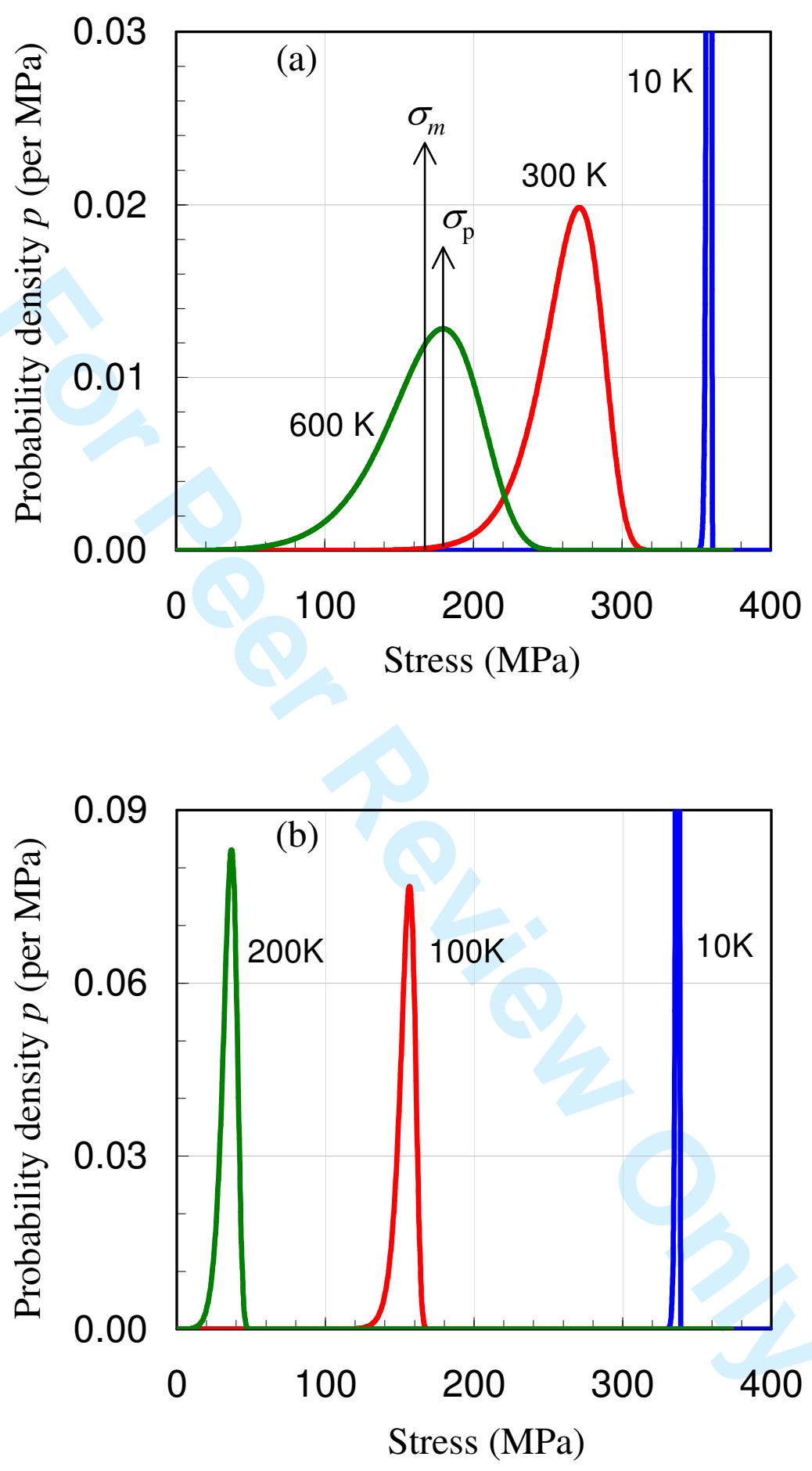

Fig. 1 


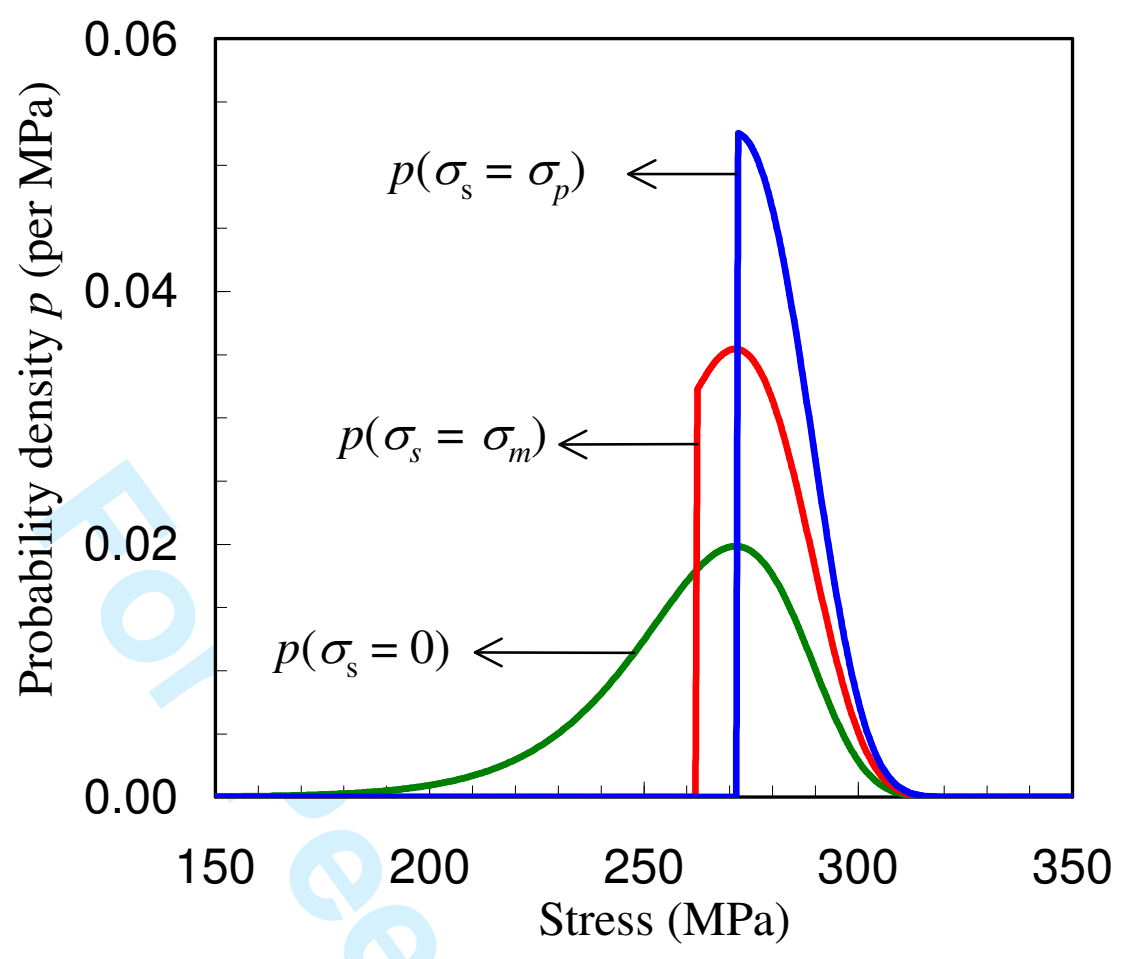

Fig. 2 

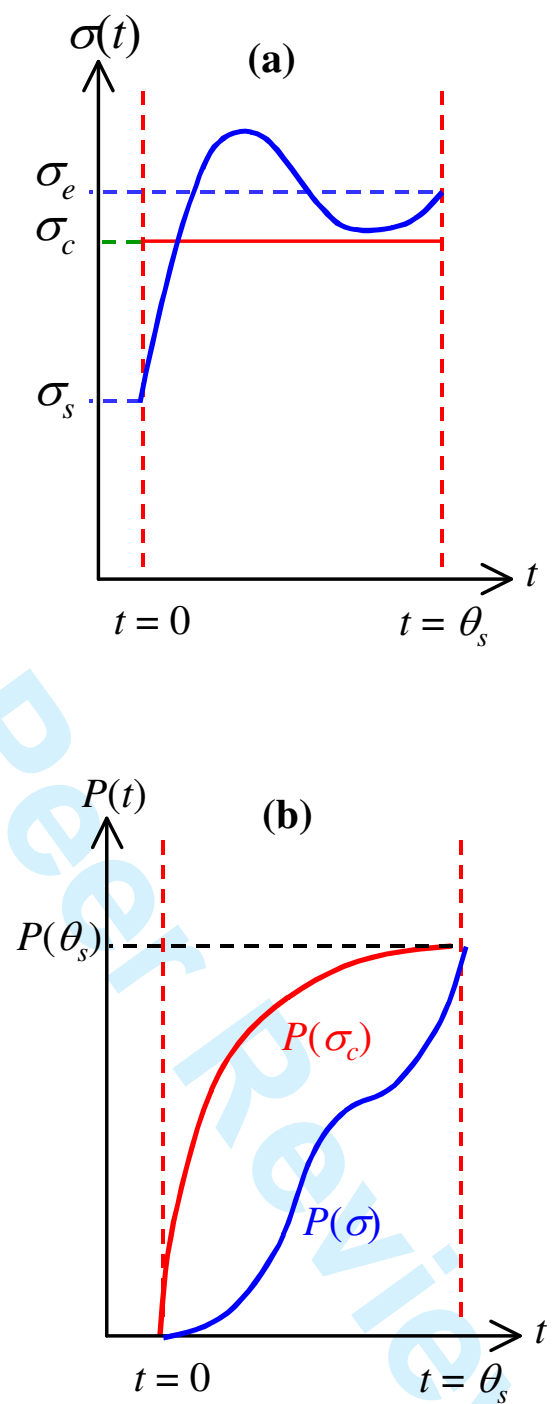

Fig. 3 


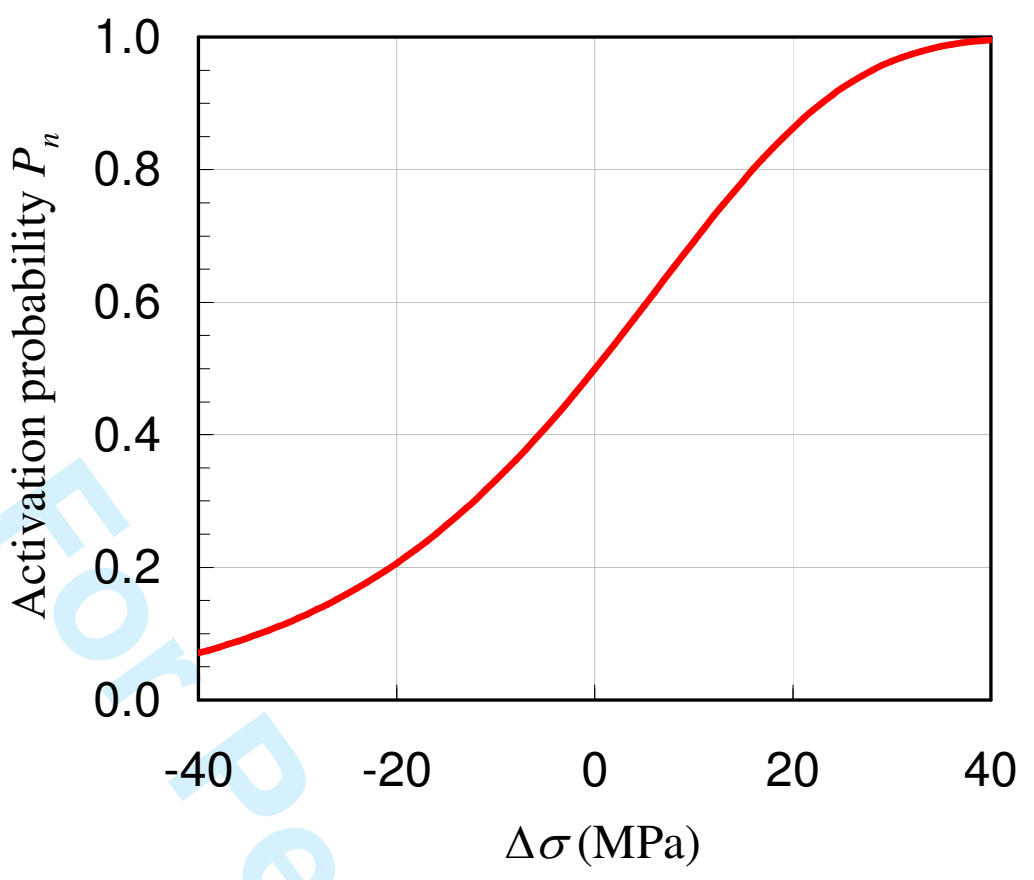

Fig. 4

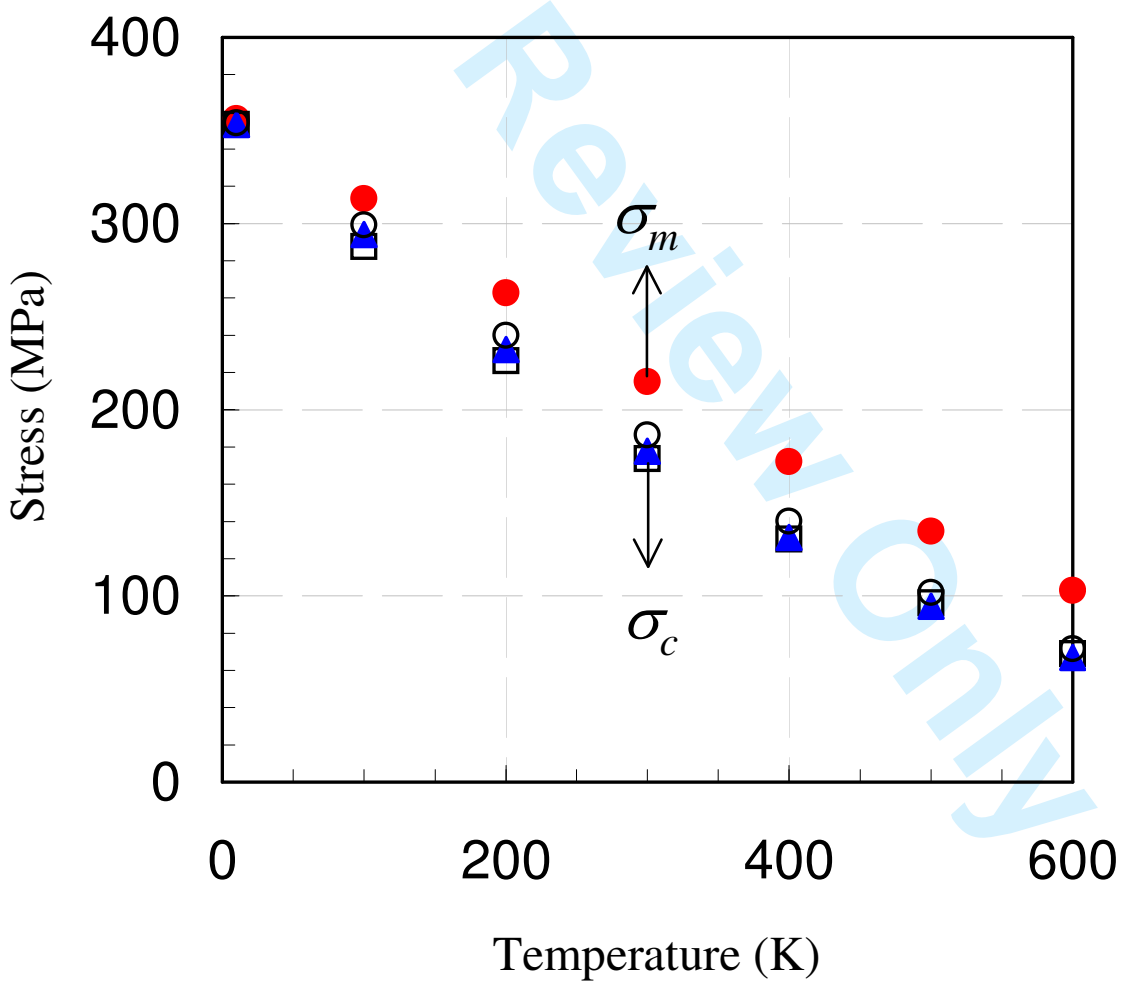

Fig. 5 


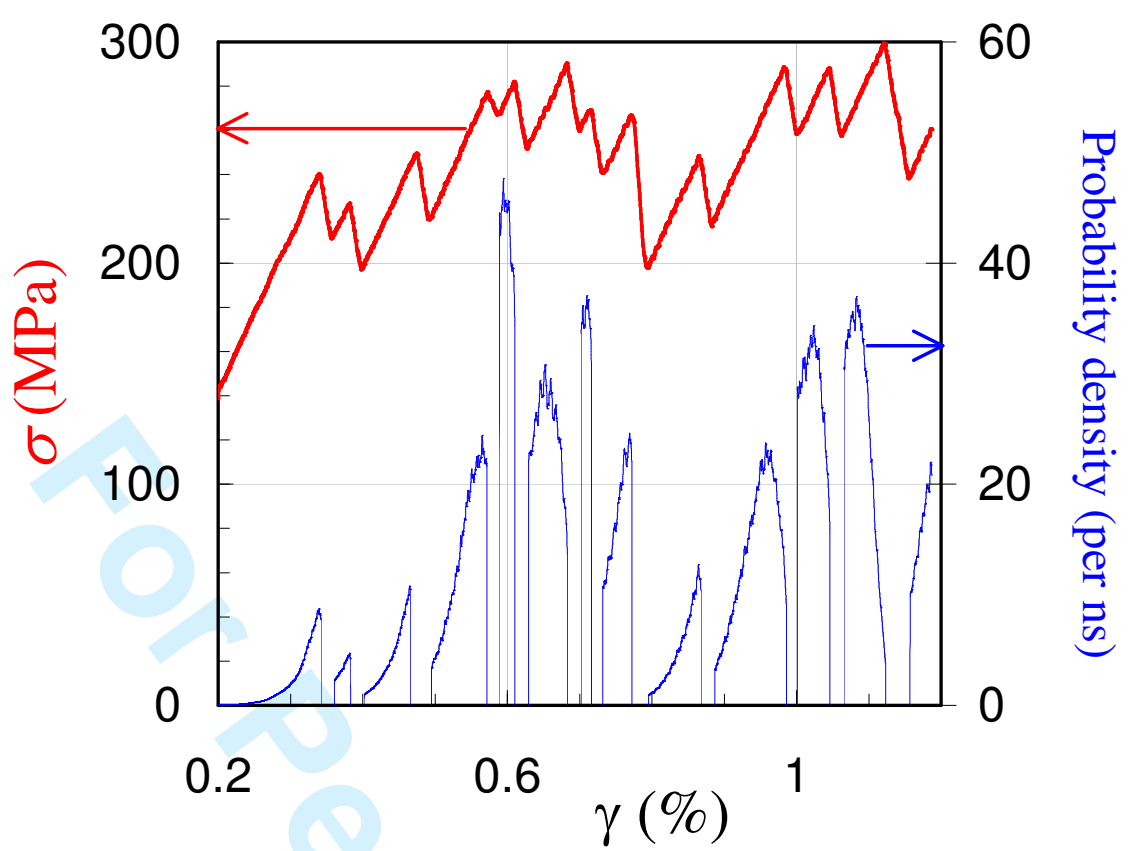

Fig. 6

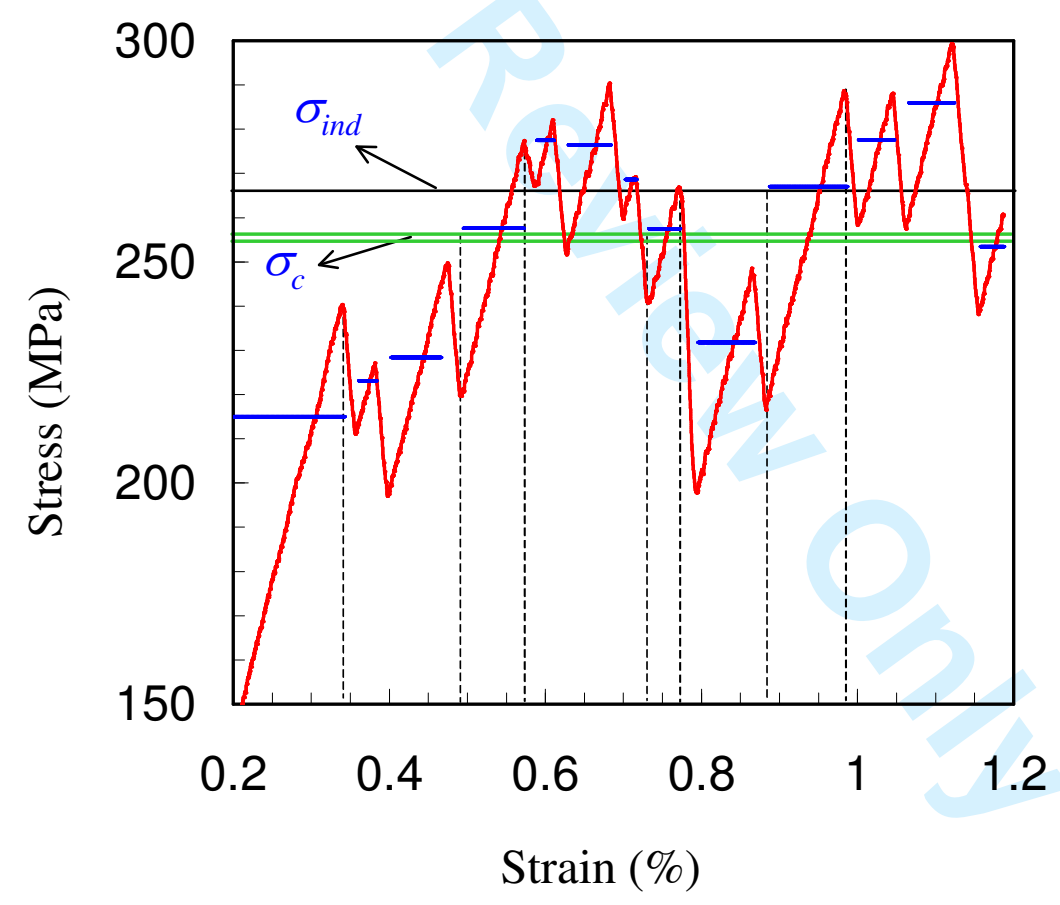

Fig. 7 


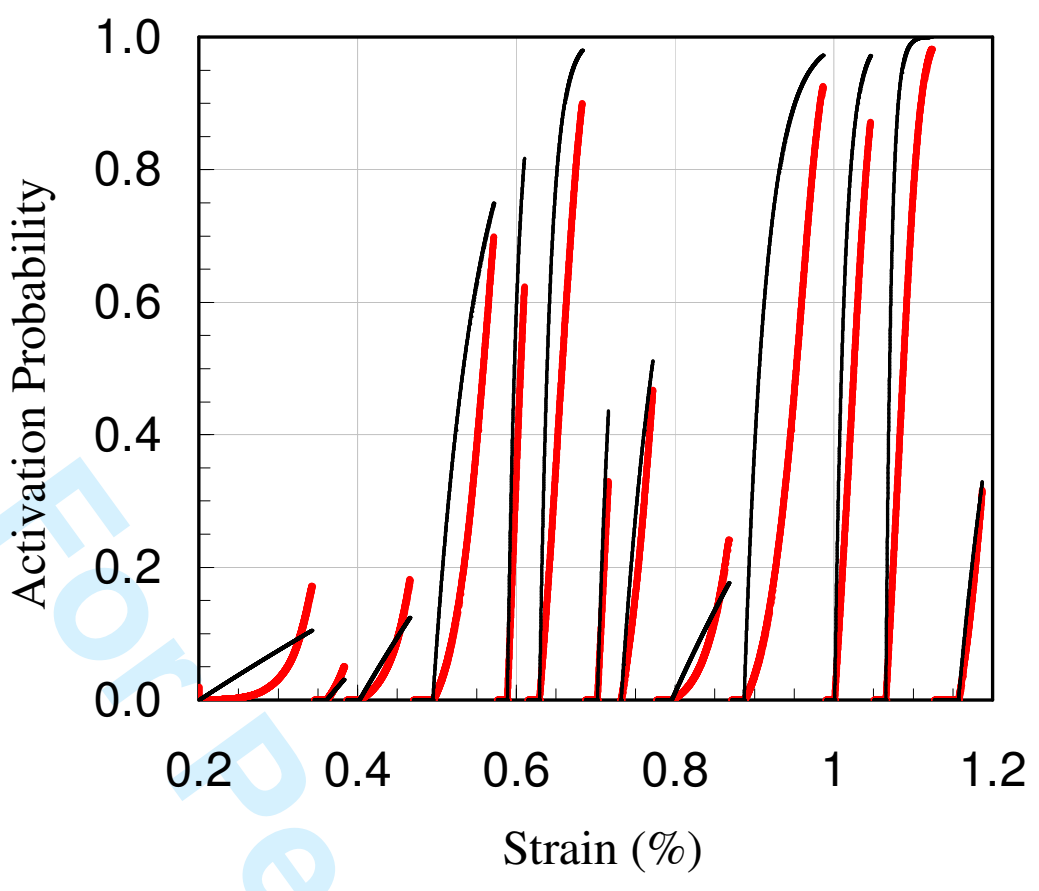

Fig. 8 